\title{
Fatores associados à sintomatologia depressiva numa coorte urbana de idosos
}

\author{
Depressive symptomatology and its associated \\ factors in an urban cohort of elderly
}

Márcio Tomita da Rocha Lima', Rebeca de Souza e Silva', Luiz Roberto Ramos'

\section{RESUMO}

Objetivo: Analisar a associação, em uma coorte urbana de idosos, entre sintomatologia depressiva e outros indicadores de capacidade funcional com a mortalidade após 15 anos de seguimento. Métodos: Foram estudados os casos de 1.667 idosos ( $\geq 65$ anos) residentes em Vila Clementino, na cidade de São Paulo, por meio de uma avaliação multidimensional da capacidade funcional e dos atestados de óbito referentes a mortes ocorridas entre 1991 (início do estudo) e abril de 2006. Na análise univariada foram calculadas as razões de chances e sua significância estatística foi avaliada pelo intervalo de confiança (95\%). Na análise múltipla foi empregada a regressão logística. Resultados: Entre os idosos estudados, 21,1\% eram deprimidos em 1991. Na análise univariada, as maiores proporções de deprimidos foram observadas entre indivíduos do sexo feminino, com mais de 80 anos, analfabetos, viúvos ou solteiros, com alto nível de dependência física e de déficit cognitivo, usuários de mais medicamentos e entre os que morreram no período de seguimento de 15 anos. Na

\section{Palavras-chave}

Depressão, capacidade funcional, idoso, mortalidade. análise múltipla, controlando-se por sexo e idade, a presença de dependência física e o fato de ser usuário de mais medicamentos foram os principais preditores da ocorrência de depressão. Conclusões: A ocorrência de sintomatologia depressiva parece estar diretamente relacionada aos níveis de incapacidade física observados entre os idosos.

\section{ABSTRACT}

Introduction: The process of population aging in Brazil has been fast and intense, increasing the prevalence of chronic diseases and psychological and mental problems, mainly depression, which is associated with functional losses of the aged one. Objective: To analyse the association between depressive symptomatology and other indicators of functional capacity, and with mortality after 15 years of follow-up, in an urban cohort of elderly. Methods: 1667 aged ( $\geq 65$ years-old) residents in Clementino Village in São Paulo city were studied through a multidimensional evaluation of the functional capacity, as well as the death certificate of the deaths occurred between 1991 (beginning of the study) and April of 2006. In univaried analysis we calculated odds ratio and its significance statistics was evaluated by the reliable interval (95\%). The logistic regression was used in the multiple analysis. Results: 21,1\% of the elderly were depressed in 1991.

1 Universidade Federal de São Paulo, Escola Paulista de Medicina (Unifesp/EPM), Departamento de Medicina Preventiva. 


\section{Keywords}

Depression, functional capacity, elderly, mortality.
In univaried analysis the highest ratios of depressed people were found in the feminine sex, aged older than 80 years-old, illiterates, widowers or bachelors, high level of physical dependence, cognitive deficit, users of more medicines and among those who died in the period of 15 years. In multivaried analysis, controlling by sex and age, physical dependence and the users of more medicines are associated to depression. Conclusions: The worse levels of disability answer, by an expressive way, to the occurrence of depressive symptomatology between the aged ones.

\section{INTRODUÇÃO}

O processo de envelhecimento populacional no Brasil tem sido rápido e intenso. A Pesquisa Nacional por Amostragem de Domicílios (PNAD), realizada em 2004 pelo Instituto Brasileiro de Geografia e Estatística (IBGE), mostra que a parcela dos brasileiros com mais de 60 anos correspondia a cerca de 10\% da população; todavia, no começo dos anos 1980, essa cifra alcançava pouco mais de $6 \%$. Notamos que a população de idosos brasileiros praticamente dobrou em 20 anos.

A resultante epidemiológica mais evidente é o aumento de doenças crônicas em idosos, bem como de incapacidades físicas e mentais associadas, geradoras de dependência no exercício das atividades diárias (Ramos et al., 1987). Com o envelhecimento da população, aumenta a prevalência de afecções psicológicas e mentais (Butler e Lewis, 1995).

É nesse contexto que a capacidade funcional, em seu sentido amplo, surge como um novo conceito de saúde. Envelhecimento saudável, nessa nova ótica, passa a ser a resultante da interação multidimensional entre saúde física, saúde mental, independência na vida diária, integração social, suporte familiar e independência econômica (Ramos et al., 1993b).

Atualmente, a depressão equivale a 1,6\% (786.000) das mortes e ao maior número de Disability Adjusted Life Years (DALY) (69.800.000) - 5,1\% do peso atribuível para condições selecionadas como fatores de risco para morte e incapacidade por outras doenças e ferimentos devem-se à depressão (Murray e Lopez, 1997).

Apesar de ser uma das condições que nas últimas décadas tem sido reconhecida mundialmente como importante e a mais comum entre as doenças mentais (Thapar, 2004), a depressão é frequentemente subdiagnosticada em nosso meio, especialmente na população de idosos; mesmo na região Sudeste do Brasil, a mais privilegiada em termos econômicos, há falhas no referido diagnóstico (Gazalle et al., 2004; Veras e Coutinho, 1994). O tratamento simples e adequado dessa morbidade, que certamente otimizaria os ganhos nesse novo conceito de saúde integral, simplesmente não ocorre, talvez por despreparo dos médicos para reconhecer a depressão no idoso.
Na verdade, o diagnóstico da depressão no idoso requer uma abordagem especial (Flint, 2004). Nessa faixa etária há uma diminuição da resposta emocional (erosão afetiva), acarretando um predomínio de sintomas como preferir ficar em casa, diminuição do sono, perda de prazer nas atividades habituais, ruminações sobre o passado e perda de energia (Gazalle et al., 2004).

Para o diagnóstico de depressão, além dos critérios do Manual Diagnóstico e Estatístico de Transtornos Mentais, $4^{\text {a }}$ edição - DSM-IV (Diagnostic and Statistical Manual of Mental Disorders) e da CID-10 (Décima Revisão da Classificação Estatística Internacional de Doenças e Problemas Relacionados à Saúde), há os instrumentos de avaliação/rastreio de saúde mental, como o SPES (Short Psychiatric Evaluation Schedule) validado para a língua portuguesa (Blay et al., 1988), e a Escala de Depressão em Geriatria - Geriatric Depression Scale, GDS (Yesavage et al., 1982). Tais instrumentos estão sendo largamente utilizados e aperfeiçoados por pesquisadores como um meio de estudar e quantificar melhor os sintomas depressivos e as doenças mentais.

Liu et al. (1997) demonstraram em estudo de uma comunidade rural chinesa, onde a atuação de médicos especialistas é muito restrita, que a prevalência de sintomatologia depressiva é o dobro do valor obtido via diagnóstico clínico e que, conforme a definição de casos, essa discrepância é ainda maior.

A prevalência de depressão varia em função das características socioeconômicas das pessoas. Mais que isso, vários são os métodos utilizados para diagnosticá-la, dificultando ainda mais a comparabilidade de resultados pontuais. Por exemplo, baseando-se em instrumentos de avaliação/rastreio, a prevalência de sintomatologia depressiva é menor em países desenvolvidos -13,5\% na Alemanha, utilizando-se o General Health Questionnaire (GHQ-12) (Papassotiropoulos et al., 1997), e 13,4\% na França, utilizando-se o Center for Epidemiological Studies Depression Scale (CES-D) (Fuhrer et al., 1992) - do que em países em desenvolvimento, como o Brasil - 35,0\% no Rio de Janeiro com o Brazil Old Age Schedule (BOAS) (Veras e Coutinho, 1994), e 36,1\% (Bourget, 1999) na periferia de São Paulo com o SPES. Entretanto, há exceções como a África do Sul, com uma prevalência de sintomatologia depressiva relativamente baixa, 13,7\% utili- 
zando-se o CARE (Comprehensive Assessment and Referral Evaluation) como instrumento (Ben-Arie et al., 1987).

A associação de depressão com outros indicadores de capacidade funcional vem sendo bastante estudada atualmente (Lenze et al., 2005). Observou-se, por exemplo, que a sintomatologia depressiva está relacionada a prejuízo cognitivo, medido pelo Mini-Exame do Estado Mental - MEEM (Stek et al., 2004), bem como a uma maior dependência nas atividades de vida diária - AVDs (Liu et al., 1997; Ormel et al., 2002; Stek et al., 2004). Vale lembrar que a depressão tem sido considerada a principal causa de anos vividos com incapacidade no mundo (Eisenberg, 1997; Murray e Lopez, 1997), sendo a quarta causa de morbidade, com a perspectiva de que suba para o segundo lugar até o ano 2025.

A sintomatologia depressiva em idosos também apresenta relação com outras doenças crônicas (Everson-Rose e Lewis, 2005) e, consequentemente, com o uso simultâneo de vários medicamentos (Iliffe et al., 1993; Rozenfeld, 2003). De fato, quase um terço dos pacientes portadores de doenças crônicas (hipertensão, acidente vascular cerebral, insuficiência cardíaca, diabetes, doença pulmonar obstrutiva crônica) sofrem de depressão (Butler e Lewis, 1995). No Brasil, particularmente, foi constatado que a depressão relacionase à mortalidade (Ramos et al., 2001). Além das causas endógenas ou psicossomáticas, algumas medicações podem causar ou simular depressão: anti-hipertensivos (propranolol, metildopa, clonidina, reserpina), corticosteroides, antipsicóticos, analgésicos, antiparkinsonianos, hormônios etc.

Em suma, há uma correlação importante entre sintomatologia depressiva e idade dos idosos, sexo feminino, analfabetismo, pobreza, uso de medicamentos, doença física, estado cognitivo e independência no dia a dia (Chong et al., 2001; Iliffe et al., 1993; Liu et al., 1997; Ormel et al., 2002; Ramos et al., 2001; Rozenfeld, 2003; Stek et al., 2004; Swenson et al., 2000).

Nesse contexto, o presente estudo tem como objetivo contribuir para o entendimento dos fatores sociodemográficos associados à depressão, bem como avaliar a associação desta morbidade com outros indicadores de capacidade funcional - função cognitiva, grau de independência nas AVDs e uso de medicamentos - e com o status de sobrevivência, 15 anos após o diagnóstico de depressão, numa coorte urbana de idosos iniciada em 1990/1991.

\section{MÉTODOS}

\section{População estudada}

A presente análise é pautada nos dados coletados pelo Projeto EPIDOSO com idosos de idade igual ou superior a 65 anos, residentes no bairro da Vila Clementino, localizado no subdistrito da Saúde, no município de São Paulo. Participaram do estudo os idosos incluídos no recenseamento dos domicílios da área (Ramos et al., 1998) e, atualmente, estimam-se 15 mil habitantes com mais de 65 anos nesse subdistrito.

O Projeto EPIDOSO, estudo de coorte de base populacional iniciado em 1991 com um total de 1.667 idosos, vigora até o presente momento e teve quatro inquéritos transversais (1991-1992; 1994-1995; 1998-1999; 2000-2001), realizados no Centro de Estudos do Envelhecimento da Universidade Federal de São Paulo/Escola Paulista de Medicina (Unifesp/ EPM). Em abril de 2006, dos 1.667 idosos iniciais, 566 haviam falecido (Ramos et al., 1998). Para a presente análise foram utilizados os resultados obtidos no primeiro levantamento domiciliar, realizado no período de 1991-1992.

Todos os sujeitos participantes da pesquisa assinaram um Termo de Consentimento Livre e Esclarecido e esta que foi aprovada pelo Comitê de Ética em Pesquisa da Universidade Federal de São Paulo.

\section{Óbitos dos idosos}

Considerando a faixa etária da população estudada, uma parcela considerável dos idosos em acompanhamento faleceu no decorrer do estudo.

As informações a respeito dos óbitos ocorridos entre 1991 e abril de 2006 foram obtidas por meio da análise dos atestados de óbito com a parceria da Fundação Sistema Estadual de Análise de Dados (SEADE). Foi também necessário fazer contato com os familiares dos pacientes que eram acompanhados no projeto para reaver, mediante a certidão de óbito, os dados necessários para o desenvolvimento da pesquisa. Com isso, acreditamos que tivemos acesso à quase totalidade dos óbitos; as raras exceções são os óbitos que, porventura, tenham ocorrido fora do Estado de São Paulo, situação supostamente infrequente e que não deve comprometer a presente análise.

\section{Avaliação dos idosos}

A avaliação dos idosos baseou-se no instrumento aplicado no primeiro inquérito domiciliar (1991-1992) denominado OARS (Older Americans Research and Services) Multidimensional Functional Assessment Questionnaire (OMFAQ), concebido nos Estados Unidos, sendo posteriormente traduzido e adaptado para o português - BOMFAQ (Brazilian Version of OMFAQ). Tal questionário fechado, já previamente utilizado em estudos transversais com residentes idosos em São Paulo (Ramos et al., 1993 a; Ramos et al., 1993b; Ramos et al., 2001), fornece dados sociodemográficos e possui instrumento para rastreamento de sintomatologia depressiva - Short Psychiatric Evaluation Schedule (Blay et al., 1988), além de avaliar a capacidade cognitiva por meio do Mini-Exame do Estado Mental (MEEM) (Folstein et al., 1975) e da independência nas atividades de vida diária - AVDs (Ramos et al., 1993a). O objetivo é proporcionar um perfil multidimensional de capacidade funcional, identificando quais as dimensões que mais direta- 
mente comprometem a capacidade funcional da população e, com isso, indicar soluções que transcendem uma linha programática, baseadas no aumento da cobertura diagnóstica e terapêutica das doenças crônicas não transmissíveis (DCNT) que acometem os idosos.

\section{Instrumento de avaliação/rastreio de saúde mental}

Para o rastreamento de sintomatologia depressiva na população estudada, utilizou-se o instrumento de avaliação/ rastreio SPES - Short Psychiatric Evaluation Schedule. Tal instrumento apresenta a vantagem de ser validado para a língua portuguesa (Blay et al., 1988), além de ser relativamente curto e de fácil treinamento. Por meio de 15 questões objetivas e diretas, explora o prazer da vida, a dificuldade do cotidiano, a disposição das pessoas, os sentimentos de felicidade e utilidade, a sensação de solidão, o sono e os sintomas físicos, como dor de cabeça, dificuldade de andar, falta de ar e fraqueza. Um escore de 7 ou mais pontos indica sintomatologia depressiva. Tem sensibilidade de $61 \%$ e especificidade de $89 \%$, sendo o valor preditivo positivo de $66 \%$ e o negativo de $87 \%$.

Os idosos foram, inicialmente, divididos em três grupos, segundo a sintomatologia depressiva: aqueles com rastreio positivo para sintomatologia depressiva tomando ou não antidepressivos - "deprimidos"; aqueles com rastreio negativo, mas que tomavam antidepressivo, ou seja, "deprimidos controlados"; e aqueles com rastreio negativo que não tomavam antidepressivos, isto é, "não deprimidos".

Todavia, a diminuta parcela de idosos com depressão controlada - menos de $2 \%$ do geral - direcionou a incluílos na categoria de deprimidos. Dessa forma, trabalharemos com a variável resposta dicotomizada em "deprimidos" e "não deprimidos".

\section{Avaliação da função cognitiva}

A função cognitiva foi avaliada por meio da versão original do "Mini-Exame do Estado Mental" (MEEM), um instrumento bastante utilizado para esse proposto. Por meio de 30 questões, avaliam-se os domínios da orientação, linguagem, memória imediata, memória de evocação, atenção e cálculo, possuindo pontuação mínima de 0 (zero) e máxima de 30 pontos/acertos. O instrumento foi originalmente validado pelo autor, com um ponto de corte de 24 pontos, em que pontuações menores identificam possível déficit cognitivo (Folstein et al., 1975). Esse ponto de corte está sujeito a um viés, caso a população tenha um nível educacional muito baixo. No caso da população em estudo, no entanto, esse viés foi minimizado pelo fato de a maioria dos respondentes ter mais de 4 anos de escolaridade. Por ser um instrumento de rastreio do comprometimento das funções cognitivas, não podemos concluir um diagnóstico de demência nos idosos sem uma avaliação neuropsicológica mais detalhada.

\section{Avaliação da independência física}

A independência nas atividades de vida diária (AVDs) foi baseada no número de AVDs para as quais se necessitava de auxílio de terceiros. Para tanto, foi utilizada uma lista com 15 itens (deitar-se/levantar-se da cama, comer, cuidar da aparência, andar no plano, tomar banho, vestir-se, ir ao banheiro em tempo, subir um lance de escada, medicar-se na hora certa, andar perto de casa, fazer compras, preparar refeições, cortar as unhas dos pés, pegar condução, fazer limpeza de casa). Foram consideradas, para cada tarefa funcional contemplada nesse instrumento, quatro categorias de respostas possíveis ("sem dificuldade", "com pouca dificuldade", "com muita dificuldade", ou "não realiza"). A seguir foi construída uma variável qualitativa com quatro categorias de dificuldade funcional, segundo o número de AVDs comprometidas: "nenhuma AVD comprometida", "1 a 3 AVDs comprometidas", "4 a 6 AVDs comprometidas" e "7 ou mais AVDs comprometidas" (Ramos et al., 1993a).

\section{Análise estatística}

Para detecção da existência de associação entre a variável resposta - deprimido ou não deprimido, no início do estudo - e cada uma das variáveis independentes, foram utilizadas razões de chances e seus respectivos intervalos de confiança (IC). Primeiramente calculou-se a chance referente a cada casela da variável resposta e, na sequência, o valor atribuído à chance da segunda, da terceira, ou das caselas subsequentes foi dividido pelo da casela de referência. Mais precisamente, no caso de a variável resposta possuir mais de duas categorias, as razões de chances e o intervalo de confiança referentes a cada uma delas foram obtidos dividindo-se a chance gerada naquela casela pela chance da primeira casela. Todos os intervalos foram calculados adotando-se um nível de significância de 5\%. Para tais cálculos foi utilizado o programa estatístico Statistical Package for the Social Sciences (SPSS).

Após realizar a análise univariada de cada uma das variáveis independentes com a variável resposta depressão, todas as variáveis que na análise univariada revelaram-se estatisticamente significante entraram no modelo completo ou saturado de regressão logística. A partir desse modelo foram retiradas, uma a uma, as variáveis que no conjunto não apresentaram associação significativa com a depressão.

\section{RESULTADOS}

Entre os 1667 idosos integrantes do Projeto EPIDOSO, em 1991, 28 não responderam às questões referentes ao instrumento SPES e, portanto, não possuíam diagnóstico para síndrome depressiva. Sendo assim, essa análise pauta-se num 
montante de 1.639 idosos, sendo que tal irrisória recusa em nada compromete os resultados aqui obtidos.

Dos 1.639 idosos em análise, 325 (19,8\%) apresentavam rastreio positivo para sintomatologia depressiva (deprimidos) e, destes, 3,7\% faziam uso de antidepressivo (deprimidos com tratamento). Apenas 21 idosos (1,3\%) apresentaram rastreio negativo tomando antidepressivo (deprimidos controlados) e, por fim, 1.293 do total de idosos $(80,2 \%)$ apresentavam rastreio negativo sem utilização de antidepressivo (não deprimidos). Como ressaltado oportunamente, para os efeitos dessa análise, enfocaremos simplesmente o grupo deprimidos (tratados ou não).

Conforme atesta a tabela 1, houve associação significante entre a sintomatologia e todas as variáveis sociodemográficas (sexo, faixa etária, escolaridade, estado civil).

Tabela 1. Distribuição dos idosos segundo variáveis sociodemográficas - Vila Clementino/São Paulo - EPIDOSO - 1991

\begin{tabular}{|c|c|c|c|c|c|c|}
\hline & \multicolumn{2}{|c|}{$\begin{array}{c}\text { Sintomatologia } \\
\text { depressiva }\end{array}$} & \multirow[t]{2}{*}{$\begin{array}{c}\text { Total } \\
\text { N }\end{array}$} & \multirow[t]{2}{*}{ Chance } & \multirow[t]{2}{*}{$\begin{array}{l}\text { Razão de } \\
\text { chances }\end{array}$} & \multirow[t]{2}{*}{ Intervalo } \\
\hline & $\begin{array}{c}\text { Deprimidos } \\
\mathrm{N}\end{array}$ & $\begin{array}{c}\text { Não } \\
\text { deprimidos } \\
\mathrm{N}\end{array}$ & & & & \\
\hline \multicolumn{7}{|l|}{ Sexo } \\
\hline Masculino & 78 & 491 & 569 & 0,16 & 1,00 & - \\
\hline Feminino & 268 & 802 & 1070 & 0,33 & 2,10 & $1,59-2,81$ \\
\hline \multicolumn{7}{|l|}{ Faixa etária } \\
\hline $65-69$ & 83 & 405 & 488 & 0,20 & 1,00 & - \\
\hline $70-74$ & 93 & 321 & 414 & 0,29 & 1,41 & $1,00-1,99$ \\
\hline $75-79$ & 77 & 304 & 381 & 0,25 & 1,24 & $0,86-1,76$ \\
\hline $80+$ & 93 & 263 & 356 & 0,36 & 1,74 & $1,23-2,47$ \\
\hline \multicolumn{7}{|l|}{ Escolaridade } \\
\hline Analfabeto & 22 & 53 & 75 & 0,42 & 1,00 & - \\
\hline Ler/escrever & 68 & 214 & 282 & 0,32 & 0,77 & $0,42-1,42$ \\
\hline Primário completo & 133 & 416 & 549 & 0,32 & 0,77 & $0,44-1,38$ \\
\hline Ginásio & 64 & 209 & 273 & 0,31 & 0,73 & $0,40-1,38$ \\
\hline Colegial/superior & 59 & 401 & 460 & 0,15 & 0,35 & $0,19-0,66$ \\
\hline \multicolumn{7}{|l|}{ Estado civil } \\
\hline Casado & 152 & 688 & 840 & 0,22 & 1,00 & - \\
\hline Divorciado & 10 & 44 & 54 & 0,23 & 1,03 & $0,45-2,13$ \\
\hline Viúvo & 145 & 451 & 596 & 0,32 & 1,46 & $1,11-1,90$ \\
\hline Solteiro & 39 & 110 & 149 & 0,35 & 1,60 & $1,04-2,44$ \\
\hline Total & 346 & 1293 & 1639 & & & \\
\hline
\end{tabular}

De fato, com respeito à variável sexo, a razão de chances mostra que entre as mulheres ocorre a maior concentração de depressão, ao assumir o valor de 2,1 variando, com 95\% de confiança, entre 1,59 e 2,81.

Quanto ao grupo etário, apenas os idosos com idade igual ou superior a 80 anos apontam comportamento estatisticamente diferente ao se tomar os mais novos - 65 a 69 anos - como referência. A razão de chances referente a tal contraste é de 1,74 e seu respectivo intervalo de confiança varia de 1,23 a 2,47.

Considerando-se, agora, o quesito escolaridade, apenas os que concluíram ao menos o colegial, atual ensino médio, apontam menor chance de ter depressão, ao se tomar os analfabetos como referência, sendo os valores da razão de chances e do intervalo de confiança de 0,35 e 0,19-0,66, respectivamente.

Para o estado civil, tomando-se a categoria de casado(a) como referência, despontam os solteiros (razão de chances $=1,60$ ) e os viúvos (razão de chances $=1,46$ ), de forma similar, concentrando maior ocorrência de depressão.

Num segundo momento, avaliamos a associação da depressão com outros indicadores de capacidade funcional - MEEM, grau de independência nas AVDs e uso de medicamentos (Tabela 2).

Tabela 2. Distribuição dos idosos segundo sintomatologia depressiva e outros indicadores de capacidade funcional - Vila Clementino/São Paulo - EPIDOSO -1991

\begin{tabular}{|c|c|c|c|c|c|c|}
\hline & \multicolumn{2}{|c|}{$\begin{array}{l}\text { Sintomatologia } \\
\text { depressiva }\end{array}$} & \multirow[t]{2}{*}{$\begin{array}{c}\text { Total } \\
\mathrm{N}\end{array}$} & \multirow[t]{2}{*}{ Chance } & \multirow[t]{2}{*}{$\begin{array}{l}\text { Razão de } \\
\text { chances }\end{array}$} & \multirow[t]{2}{*}{ Intervalo } \\
\hline & $\begin{array}{c}\text { Deprimidos } \\
\text { N }\end{array}$ & $\begin{array}{c}\text { Não } \\
\text { deprimidos } \\
\text { N }\end{array}$ & & & & \\
\hline \multicolumn{7}{|l|}{ Escore MEEM } \\
\hline $24+$ & 209 & 951 & 1160 & 0,22 & 1,00 & - \\
\hline$<24$ & 137 & 342 & 479 & 0,40 & 1,81 & $1,41-2,33$ \\
\hline \multicolumn{7}{|l|}{ AVDs comprometidas } \\
\hline Independente & 40 & 506 & 546 & 0,08 & 1,00 & - \\
\hline $1-3$ & 89 & 460 & 549 & 0,19 & 2,45 & $1,63-3,73$ \\
\hline $4-6$ & 86 & 185 & 271 & 0,46 & 5,88 & $3,83-9,10$ \\
\hline $7+$ & 120 & 125 & 245 & 0,96 & 12,14 & $7,93-18,71$ \\
\hline \multicolumn{7}{|l|}{ Medicamentos em uso } \\
\hline Nenhum & 30 & 344 & 374 & 0,09 & 1,00 & - \\
\hline $10 u 2$ & 145 & 584 & 729 & 0,25 & 2,85 & $1,86-4,47$ \\
\hline 3 ou 4 & 87 & 262 & 349 & 0,33 & 3,81 & $2,40-6,16$ \\
\hline $5+$ & 82 & 103 & 185 & 0,80 & 9,13 & $5,56-15,14$ \\
\hline \multicolumn{7}{|l|}{ Mortalidade } \\
\hline Não & 209 & 882 & 1091 & 0,17 & 1,00 & - \\
\hline $\operatorname{Sim}$ & 137 & 411 & 548 & 0,33 & 1,41 & $1,09-1,81$ \\
\hline
\end{tabular}

Associação significante foi observada entre a sintomatologia depressiva e o estado cognitivo, medido pelo MEEM. Os que apresentaram comprometimento do estado cognitivo (escore no MEEM < 24) eram mais deprimidos, sendo a razão de chances de 1,81 (IC: 1,41 - 2,33) com respeito àqueles sem déficit cognitivo (escore no MEEM > ou igual a 24).

Com relação ao comprometimento nas atividades de vida diária, verifica-se uma tendência crescente na relação de deprimidos, ao se passar do grupo de independentes a cada um dos outros grupos com alguma dependência. A razão de chances para o grupo de 1 a 3 AVDs comprometidas é de 2,45; para o grupo de 4 a 6 AVDs é de 5,88; e assume a elevada cifra de 12,14 para o grupo com 7+ AVDs todos têm os independentes como referência. Todas essas razões de chances estão associadas a IC que não contêm a unidade, sendo, portanto, todos significativamente mais elevados que o da casela de referência.

Entre os idosos, 54\% utilizavam algum medicamento cardiovascular, 21\%, algum medicamento relacionado ao trato alimentar e metabolismo e 18\%, drogas para o sistema nervoso. Ao analisarmos a relação entre o número de me- 
dicamentos tomados pelos idosos participantes do estudo e a sintomatologia depressiva, também foi observada uma tendência crescente na proporção de deprimidos, sempre estatisticamente significante, ao aumentar a quantidade de medicamentos utilizados. Os que tomavam entre 1 ou 2 revelaram uma razão de chances de 2,85 diante dos que não usavam qualquer medicação; para os que consumiam 3 ou 4, essa razão de chances foi de 3,81; e foi de 9,13, para os que utilizavam 5 ou mais medicamentos.

Dos 566 idosos falecidos, desde 1991 até abril de 2006, 548 haviam respondido ao SPES no início do estudo e foram, assim, incluídos na análise. Nesta, foi observada, por fim, associação estatisticamente significante entre sintomatologia depressiva e mortalidade em 2006. Dentre os 548 óbitos válidos, 25,0\% eram deprimidos no início do estudo, sendo a razão de chances de 1,41 (IC: 1,09-1,81) tomandose como referência aqueles que permaneciam vivos até abril de 2006. Entretanto, o limite inferior desse intervalo de confiança é mais próximo do valor unitário que qualquer um dos demais que revelaram significância estatística.

Agregando-se a tal achado a informação de que a idade média ao morrer, entre os deprimidos e os não deprimidos, não difere estatisticamente $(p=0,16)$, sendo de 82,76 anos $(\mathrm{DP}=8,18)$ e de 81,25 anos ( $\mathrm{DP}=7,39)$, na ordem citada, constata-se que a depressão tem um efeito independente da idade na mortalidade.

Na sequência, foi rodado o modelo de regressão completo com todas as variáveis que apresentaram associação significante com depressão (Tabelas 1 e 2). Retirando-se, uma a uma, as variáveis que produzem um valor de $p$ - probabilidade de rejeitar $\mathrm{Ho}>0,05$ - restaram no modelo final as variáveis: sexo, idade, número de medicamentos em uso e grau de independência nas atividades de vida diária.

Observa-se pela tabela 3 que, controlando-se pelas variáveis sexo e idade, a razão de chances referente ao número de medicamentos em uso pelos idosos é de 1,44 e o referente ao grau de independência nas AVDs é de 2,12. Portanto, mediante o número de medicamentos em uso, o grau de dependência nas AVDs na análise conjunta responde de forma mais expressiva à ocorrência de depressão.

Tabela 3. Modelo final de regressão logística* para sintomatologia depressiva - Vila Clementino/São Paulo - EPIDOSO - 1991

\begin{tabular}{lcc}
\hline Variáveis & Razão de chances & $\boldsymbol{p}$ \\
\hline Independência física (AVDs) & 2,12 & $<0,001$ \\
Uso de medicamentos & 1,44 & $<0,001$ \\
Constante & 0,018 & $<0,001$ \\
\hline
\end{tabular}

${ }^{*}$ Controlado por sexo e idade.

\section{DISCUSSÃO}

Em nossa coorte de idosos verificamos, no início do estudo em 1991, prevalência de sintomatologia depressiva de 21,1\%, número que se aproxima bastante daqueles observados em países desenvolvidos (Fuhrer et al., 1992; Papassotiropoulos et al., 1997) e bem inferior aos 38,5\% observados em estudo recente com idosos residentes em Bambuí, uma comunidade do interior de Minas Gerais no Sudeste do Brasil (Castro-Costa et al., 2008). O fato de a prevalência encontrada se aproximar dos números dos países mais desenvolvidos pode ter ocorrido devido aos idosos em estudo residirem no subdistrito da Saúde do município de São Paulo, área que possui características socioeconômicas melhores que a média do país.

Ao associarmos a sintomatologia depressiva com diferentes variáveis sociodemográficas no início do estudo, pudemos observar maior prevalência de sintomatologia depressiva no sexo feminino, nos muito idosos (80+), e entre solteiros ou viúvos. O fato de ter cursado colegial ou nível superior demonstrou ser fator de proteção para sintomatologia depressiva. Tais resultados são similares aos encontrados em outros estudos (Chong et al., 2001; Liu et al., 1997; Ramos et al., 2001; Swenson et al., 2000). Entretanto, os mesmos resultados vão de encontro aos observados em estudo realizado recentemente no estado do Rio Grande do Sul - Brasil, no qual não foi verificada diferença significativa entre os sexos ou o grau de escolaridade dos idosos (Blay et al., 2007).

A prevalência de depressão foi significativamente maior entre os idosos com a função cognitiva prejudicada (escore MEEM < 24) do que entre os idosos sem déficit cognitivo, assinalando uma associação de dois diagnósticos bastante comuns entre idosos - depressão e déficit cognitivo. Ambos estão perversamente inter-relacionados, já que a depressão pode muitas vezes levar a quadros clínicos diagnosticados como demência e casos de demência geralmente cursam com sintomas de depressão.

Ademais, também foi observada tendência crescente na proporção de deprimidos à medida que os idosos se tornavam mais dependentes fisicamente. Há uma maior proporção de deprimidos entre os idosos com maiores índices de comprometimento nas AVDs.

O número de medicamentos em uso também apresentou forte relação com a sintomatologia depressiva, refletindo sua associação com doenças crônicas, e sendo tal resultado compatível com o observado em uma pesquisa realizada na Inglaterra (lliffe et al., 1993) e em um estudo brasileiro (Rozenfeld, 2003).

A sintomatologia depressiva aumentou marginalmente o risco de morte sem, no entanto, afetar a idade média ao morrer. Por estar fortemente associada com os piores níveis de capacidade funcional que, por sua vez, aumenta em muito o risco de morte (Ramos et al., 2001), a sintomatologia depressiva pode não ser um fator determinante de mortalidade mas certamente contribuiu para a perda funcional do idoso.

Os dados obtidos contribuem para que os profissionais da área da saúde tenham conhecimento do perfil do idoso 
deprimido e da íntima relação da sintomatologia depressiva com maiores prejuízos da capacidade funcional, seja física ou mental. A julgar pela pequena porcentagem de deprimidos tratados encontrada no estudo, podemos supor que a depressão na população idosa seja subdiagnosticada e subtratada.

Levando-se em conta a forte associação de depressão com incapacidades, independentemente do sexo e da idade, devemos considerá-la um importante parâmetro da saúde dos idosos. Cabe ressaltar a necessidade de um maior cuidado para com o idoso deprimido. Sendo assim, medidas visando manter o idoso ativo e inserido na sociedade, como a implementação de Centros de Convivência do Idoso, poderiam auxiliar na prevenção da síndrome depressiva.

\section{AGRADECIMENTOS}

A Franklin Massanori Amaya pela oportunidade de estudar o envelhecimento. A Vera de Fátima da C. R. Souza e Valdirene Aparecida da Silva Gomes pelo auxílio com a informação sobre os óbitos dos pacientes. A Samuel Ponce pela ajuda na computação dos dados. Trabalho financiado pela Fundação de Amparo à Pesquisa do Estado de São Paulo (Fapesp) (\# 90/3935-7), e pelo Conselho Nacional de Desenvolvimento Científico e Tecnológico (CNPq) por meio do Programa de Bolsas de Iniciação Científica (PIBIC) (\# 103133/2004-6).

\section{REFERÊNCIAS}

1. Ben-Arie 0, Swartz L, Dickman BJ. Depression in the elderly living in the community. Its presentation and features. Br J Psychiatry. 1987;150:169-74.

2. Blay SL, Andreoli SB, Fillenbaum GG, Gastal FL. Depression morbidity in later life: prevalence and correlates in a developing country. Am J Geriatr Psychiatry. 2007;15:790-9.

3. Blay SL, Ramos LR, Mari J. Validity of a Brazilian version of the Older Americans Resources and Services (OARS) mental health screening questionnaire. J Am Geriatr Soc. 1988;36(8):687-92.

4. Bourget M. Prevalência de sintomatologia relacionada à depressão entre idosos de baixa renda, na periferia do Município de São Paulo [tese de mestrado]. São Paulo: Universidade Federal do Estado de São Paulo; 1999.

5. Butler RN, Lewis MI. Late-life depression: when and how to intervene. Geriatrics. 1995;50(8):44-6,49-52,55;quiz 56.

6. Castro-Costa E, Lima-Costa MF, Carvalhais S, Firmo J0, Uchoa E. Factors associated with depressive symptoms measured by the 12-item General Health Questionnaire in communitydwelling older adults (The Bambuí Health Aging Study). Rev Bras Psiquiatr. 2008;30:104-9.

7. Chong MY, Tsang HY, Chen CS, Tang TC, Chen CC, Yeh TL, et al.. Community study of depression in old age in Taiwan: prevalence, life events and socio-demographic correlates. Br J Psychiatry. 2001;178(1):29-35.
8. Eisenberg L. Psychiatry and health in low-income populations. Compr Psychiatry. 1997; 38(2):69-73.

9. Everson-Rose SA, Lewis TT. Psychosocial factors and cardiovascular diseases. Annu Rev Public Health. 2005;26:469-500.

10. Flint AJ. Epidemiology and comorbidity of anxiety disorders in the elderly. Am J Psychiatry. 1994;151(5):675-80. Review.

11. Folstein MF, Folstein SE, McHugh PR. "Mini-mental state". A practical method for grading the cognitive state of patients for the clinician. J Psychiatr Res. 1975;12(3):189-98.

12. Fuhrer R, Antonucci TC, Gagnon M, Dartigues JF, Barberger-Gateau P, Alperovitch A. Depressive symptomatology and cognitive functioning: an epidemiological survey in an elderly community sample in France. Psychol Med. 1992;22(1):159-72.

13. Gazalle FK, Hallal PC, Lima MS. Depression in the elderly: are doctors investigating it? Rev Bras Psiquiatr. 2004;26(3):145-9.

14. Iliffe S, Tai SS, Haines A, Booroff A, Goldenberg E, Morgan P, et al. Assessment of elderly people in general practice. 4. Depression, functional ability and contact with services. Br J Gen Pract. 1993;43(374):371-4.

15. Lenze EJ, Schulz R, Martire LM, Zdaniuk B, Glass T, Kop WJ, et al. The course of functional decline in older people with persistently elevated depressive symptoms: Iongitudinal findings from the Cardiovascular Health Study. J Am Geriatr Soc. 2005;53(4):569-75.

16. Liu CY, Wang SJ, Teng EL, Fuh JL, Lin CC, Lin KN, et al. Depressive disorders among older residents in a Chinese rural community. Psychol Med. 1997;27(4):943-9.

17. Murray CJ, Lopez AD. The utility of DALYs for public health policy and research: a reply. Bull World Health Organ. 1997;75(4):377-81.

18. Ormel J, Rijsdijk FV, Sullivan M, van Sonderen E, Kempen GI. Temporal and reciprocal relationship between IADL/ADL disability and depressive symptoms in late life. J Gerontol B Psychol Sci Soc Sci. 2002;57(4):338-47.

19. Papassotiropoulos A, Heun R, Maier W. Age and cognitive impairment influence the performance of the General Health Questionnaire. Compr Psychiatry. 1997;38(6):335-40.

20. Ramos LR, Veras $R$, Kalache A. Populational ageing: a Brazilian reality. Rev Saúde Pública. 1987;21:211-24

21. Ramos LR, Perracini M, Rosa TEC, Kalache A. Significance and management of disability among urban elderly residents in Brazil. J Cross Cultural Gerontol. 1993;8:313-23.

22. Ramos LR, Rosa TEC, Oliveira ZM, Medina MCG, Santos FRG. Profile of the elderly in an metropolitan area of Southeastern Brazil: results of a household survey. Rev Saúde Pública. 1993;27(2):87-94

23. Ramos LR, Toniolo J, Cendoroglo MS, Garcia JT, Najas MS, Perracini M, et al. Two-year followup study of elderly residents in S. Paulo, Brazil: methodology and preliminary results. Rev Saúde Pública. 1998;32(5):397-407.

24. Ramos LR, Simoes EJ, Albert MS. Dependence in activities of daily living and cognitive impairment strongly predicted mortality in older urban residents in Brazil: a 2-year follow-up. J Am Geriatr Soc. 2001;49(9):1168-75.

25. Rozenfeld $S$. Prevalence, associated factors, and misuse of medication in the elderly: a review. Cad Saúde Pública. 2003;19(3):717-24.

26. Stek ML, Gussekloo J, Beekman AT, van Tilburg W, Westendorp RG. Prevalence, correlates and recognition of depression in the oldest old: the Leiden 85-plus study. J Affect Disord. 2004;78(3):193-200

27. Swenson CJ, Baxter J, Shetterly SM, Scarbro SL, Hamman RF. Depressive symptoms in Hispanic and non-Hispanic white rural elderly: the San Luis Valley Health and Aging Study. Am J Epidemiol. 2000;152(11):1048-55.

28. Thapar GD. Approach to the problems of the aged. J Indian Med Assoc. 2004;102(2):93-6.

29. Veras RP, Coutinho E. Prevalence of organic brain syndrome in an elderly population in a metropolitan population of southeastern Brazil. Rev Saúde Pública. 199428(1):26-37.

30. Yesavage JA, Brink TL, Rose TL, Lum 0, Huang V, Adey M, et al. Development and validation of a geriatric depression screening scale: a preliminary report. J Psychiatr Res. 19821983;17(1):37-49. 